\title{
Factores que Afectan el Desempeño Docente en Centros de Alta y Baja Eficacia en México
}

\author{
Factors that Affect Teaching Performance in High and Low \\ Effectiveness Schools in Mexico
}

\author{
Rubí Peniche Cetzal 1* \\ Cristóbal Ramón Mac ${ }^{1}$ \\ Cintya Guzmán Ramírez ${ }^{2}$ \\ Noé Mora Osuna ${ }^{1}$ \\ ${ }^{1}$ Universidad Autónoma de Aguascalientes, México \\ ${ }^{2}$ Instituto de Educación de Aguascalientes, México
}

\begin{abstract}
El estudio se fundamenta en la perspectiva de la eficacia escolar, donde se analizan los factores que afectan el desempeño del docente de enseñanza secundaria, desde la opinión de directivos escolares, en el estado de Aguascalientes (México). Se realizó un estudio mixto, el enfoque cuantitativo que permitió identificar y seleccionar a las escuelas de alta y baja eficacia escolar, mediante el desarrollo de modelos jerárquicos lineales; el enfoque cualitativo consistió en entrevistar a directivos de las escuelas seleccionadas. Los resultados indican la existencia de factores internos y externos. Dentro de los factores internos es el compromiso docente el que marca diferencias entre las escuelas de alta y baja eficacia, al ser característico de los profesores del primer tipo de centro. Los factores externos como los recursos y materiales, la estabilidad laboral y la carga horaria afectan la participación y compromiso de los docentes en los objetivos y proyectos institucionales. Los resultados y conclusiones son consistentes con estudios previos y se considera que este trabajo es una puerta a estudios que permitan profundizar al interior de las aulas, de tal forma que sea posible identificar información más específica.
\end{abstract}

Descriptores: Eficacia escolar; Eficacia del docente; Director de colegio; Enseñanza secundaria; Innovación educacional.

This paper is based on school effectiveness perspective, which analyzes the factors that, affect the performance of the teacher of secondary education, from the opinion of the school principals, in the state of Aguascalientes (Mexico). A mixed study was conducted, first a quantitative approach which identified high and low efficiency schools, through the development of linear hierarchical models; the qualitative approach consisted of conducting semi-structured interviews with principals of the selected schools. The results indicate that, based on the opinion of the school principals, within the internal factors it is the teaching commitment that marks differences between high and low efficiency schools, being characteristic of the teachers of the first type of school. Likewise, external factors such as resources and materials, work stability and the workload significantly affect the teacher's participation and commitment about the school goals and institutional projects and, consequently, the achievement of the students and the functioning of the schools. The results and conclusions are consistent with previous studies and this work a door to studies that allow deepening inside the classrooms of effective and noneffective schools, in such a way that it is possible to identify information that is more specific.

Keywords: School effectiveness; Teacher effectiveness; Head teacher; Secondary education; Educational innovations.

*Contacto: rupeniche81@gmail.com

ISSN: $1696-4713$

www.rinace.net/reice/

revistas.uam.es/reice
Recibido: $\quad 21$ de octubre 2019

$1^{\text {a }}$ Evaluación: 25 de diciembre 2019

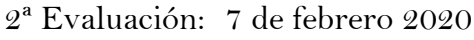

Aceptado: 18 de febrero 2020 


\section{Introducción}

En el Sistema Educativo Mexicano la Educación Media Superior (EMS, equivalente a la Educación Secundaria, $2^{\circ}$ nivel), forma parte de la educación obligatoria desde el año 2012 (Diario Oficial de la Federación, 2012), y se tiene la meta de alcanzar su universalización para el ciclo escolar 2021-2022 (Tuirán y Hernández, 2016). Este tipo educativo atiende en su mayoría a jóvenes en edad normativa de entre 15 y 17 años y comprende tres modelos educativos: general, tecnológico y profesional técnico.

De acuerdo con datos del Instituto Nacional para la Evaluación de la Educación (INEE, 2018), durante el ciclo escolar 2016-2017, la matrícula de la EMS era equivalente a 16,6\% (5.128.518) de los estudiantes en el país (30.909.211). En ese mismo ciclo escolar, estaba integrado por 17.723 planteles (7,3\% del total nacional), y contaba con los servicios de 298,335 docentes (19,7\%). En el estado de Aguascalientes, se cuenta con 309 escuelas y 3,995 docentes, quienes atienden a 61,301 estudiantes (IEA, 2017).

En México, este tipo educativo enfrenta algunos desafíos; entre estos, Tuirán y Hernández (2016) reconocen: ampliar las oportunidades para el ingreso; trabajar en la equidad y la inclusión; combatir el abandono escolar y apoyar la continuidad de las trayectorias educativas; atender la profesionalización docente, los aprendizajes, la infraestructura y el equipamiento de los planteles; abordar la prevalencia de conductas de riesgo entre los estudiantes y promover la articulación de los perfiles de egreso con la demanda laboral. Por su parte, Ruiz y Luna (2017) explican que, como consecuencia de las problemáticas enunciadas, actualmente se enfrentan serias dificultades para conseguir la meta de la universalización para el ciclo escolar 2021-2022.

Respecto de los retos en materia de equidad e inclusión, Tuirán y Hernández (2016) explican que, en México, los sectores de la población ubicados en los estratos socioeconómicos menos favorecidos suelen ser los que conjuntan un menor porcentaje de estudiantes inscritos en este tipo educativo, además de que el abandono escolar y la falta de continuidad de los estudios son el reflejo de la exclusión educativa. Al respecto, el INEE (2017) indica que 30,0 \% de los jóvenes en edad normativa para cursar la EMS no tiene acceso a ella, además, únicamente $70,0 \%$ de aquellos que ingresan consiguen finalizar sus estudios en el tiempo reglamentario, que es de tres años.

En México, acerca de los aprendizajes alcanzados al finalizar la educación obligatoria, los datos también reflejan problemáticas. De acuerdo con Backhoff, Guevara, Hernández y Sánchez (2018) los resultados obtenidos en el Plan Nacional para la Evaluación de los Aprendizajes (PLANEA/EMS) indican que 33,9 \% de los jóvenes se ubican en el Nivel I (el más bajo) en el área de lenguaje y comunicación, contrastando con 9,2 \% en Nivel IV (el más alto). La situación se agudiza en el área de matemáticas, donde sólo 2,5\% de los jóvenes se ubicaron en el Nivel IV y 66,2 \% en el Nivel I. Los autores agregan que las diferencias entre los estudiantes con las puntuaciones más altas y aquellos con las más bajas corresponden a más de dos grados escolares de desempeño en ambas áreas.

En lo que respecta a la profesionalización docente, los datos disponibles muestran que, $65,5 \%$ de los maestros de este tipo educativo cuentan con licenciatura terminada, 12,3\% con maestría y únicamente $0,87 \%$ con doctorado (INEE, 2015). Este mismo organismo reconoce que, ante la disparidad en cuanto a la profesionalización, los cursos de inducción y de actualización son esenciales para enriquecer la formación pedagógica con la que 
ingresan los docentes a este nivel educativo, especialmente si se considera que varios de ellos no cuentan con formación pedagógica.

En cuanto a los factores laborales, el INEE (2015) reportó que el tipo de contratación de los docentes de EMS en el país es principalmente por horas, de tal forma que 66,0 \% de aquellos provenientes de bachilleratos generales está en esta condición, así como 76,0 \% del profesional técnico. Entre ambas modalidades reúnen sólo 13,0\% de docentes contratados por tiempo completo, situación que mejora en los bachilleratos tecnológicos con $24,0 \%$. Estas condiciones pueden dificultar el trabajo colegiado entre los docentes, así como la posibilidad de participar en actividades escolares, o destinar mayor tiempo a actividades de planeación o incluso de profesionalización (Tuirán y Hernández, 2016).

Ante la necesidad de formular opciones que contribuyan a mejorar los resultados educativos de los jóvenes durante su tránsito por la educación obligatoria, en este estudio se reconoce la participación de distintos actores como son: los padres de familia, los directivos, los estudiantes y los docentes. Estos últimos mantienen un papel fundamental, al ser ellos quienes se encuentran al interior de las aulas y de quienes depende la mayor parte del proceso educativo, razón por la cual la atención de este trabajo se centra en esta figura y en lo que realiza en el nivel aula, que para fines de este escrito será estudiado desde la opinión de los directivos, pues estos son piezas clave en la búsqueda de la eficacia y mejora escolar, al tener la responsabilidad de facilitar las condiciones para generar una educación de calidad (Scheerens, 2016). Además, debe desempeñar un liderazgo educativo que facilite el trabajo colaborativo, la participación de los distintos actores, la evaluación continua, entre otras funciones (Secretaría de Educación Pública [SEP], 2012). Por lo anterior, es considerado un informante clave para lograr un primer acercamiento a lo que ocurre al interior de los centros escolares y de las aulas, pues son quienes tienen la responsabilidad de conocer, comunicar y coordinar lo que ocurre en las escuelas.

Se presentan a continuación los resultados de un estudio desarrollado con el objetivo general de identificar los factores que afectan el trabajo del docente de EMS en escuelas de alta y baja eficacia escolar, desde la perspectiva del directivo. Además, se plantearon dos objetivos específicos:

- Identificar los factores externos e internos al profesor de EMS que afectan el trabajo que realiza al interior de la escuela y del aula.

- Realizar aportaciones al estudio de escuelas eficaces que permitan validar resultados de estudios previos, así como abran la puerta a nuevos trabajos de investigación en esta materia.

Los hallazgos que se reportan corresponden a un estudio más amplio titulado Modelo comprehensivo de la mejora de la eficacia escolar en educación media superior en el estado de Aguascalientes (México).

Se tomó la decisión de fundamentar el estudio en la perspectiva de la eficacia escolar, ya que reconoce la capacidad de las escuelas para influir en los logros escolares y sociales de los estudiantes (Chapman et al., 2016). Asimismo, esta perspectiva postula que las acciones que el docente desarrolla al interior del aula se encuentran fuertemente relacionadas con los logros de los alumnos; por lo tanto, el presente trabajo explora un conjunto de factores relativos al docente, mismos que fueron divididos en: (a) internos, aquellos que son competencia de los docentes (compromiso docente); y (b) externos, que son responsabilidad de la escuela o del sistema educativo (clima organizacional, dotación de 
materiales y recursos para la enseñanza, capacitación y actualización, estabilidad laboral y carga horaria).

\section{Escuelas eficaces: El trabajo del docente}

La investigación en eficacia escolar se enfoca en establecer qué hace a una escuela obtener mejores resultados que otras en condiciones similares, o qué le permite a la escuela dar un valor añadido a sus estudiantes, así como describir los factores escolares internos (en particular) y del sistema educativo (en general) que podrían afectar los resultados de aprendizaje de los estudiantes, tanto en el ámbito académico como en el social (Chapman et al., 2016). Con lo anterior, ha de entenderse que la eficacia escolar se refiere al impacto que los factores escolares tienen en el desempeño académico y afectivo de los alumnos, como lo plantean Creemers y Kyriakides (2008).

En el contexto iberoamericano, la investigación realizada en varios países por medio del Convenio Andrés Bello (Murillo, 2007) dio como resultado la propuesta de un Modelo Iberoamericano de Eficacia Escolar. El estudio partió de una visión multinivel y sistémica de la educación, al considerar cuatro niveles -y sus correspondientes factores-: alumno, aula, escuela y sistema educativo, y que cada nivel está inserto en otro y por ello, están interrelacionados. Los resultados indicaron que los factores de aula tienen efectos importantes en el logro académico cognitivo en matemáticas y lengua (22,0 \% y 11,0 \% de varianza, respectivamente) y más aún en el logro académico socio-afectivo en cuanto a convivencia social, satisfacción con la escuela, comportamiento y autoconcepto (con 35,2 $\%, 24,2 \%, 10,59 \%$ y $10,0 \%$ de varianza, en ese orden).

Entre los factores de aula que inciden en el rendimiento se encuentran: características del aula, características del docente, metodología docente, gestión del tiempo, clima de aula, expectativas del docente hacia sus alumnos, compromiso del docente, formación permanente del profesorado e instalaciones y recursos. Se puede observar que, gran parte de estos factores corresponden al trabajo que debe desempeñar el docente o a características propias de éste, por lo que se puede establecer que su papel es determinante en los resultados que se obtienen en el nivel de aula, como lo afirman Davis y Thomas (2007):

\footnotetext{
[...] no son ni el director ni la escuela quienes se enfrentan todos los días con los alumnos, ni siquiera quienes les enseñan; son los profesores. Los profesores pueden dirigir con o sin fortuna a sus alumnos hacia mayores niveles de aprendizaje y hacia más altas aspiraciones. (p. 27)
}

En sintonía con lo expresado, Martínez-Garrido y Murillo (2016), afirman que es importante investigar cómo se desarrollan las actividades en las aulas de las escuelas eficaces, dado que los procesos que se realizan en las primeras corresponden a los elementos que más inciden en el progreso académico de los alumnos. En el mismo sentido lo expresa Scheerens (2016), en cuanto a que las variables más próximas al aprendizaje del estudiante tienen un mayor impacto que las variables más distantes. A partir de lo anterior, reviste gran importancia identificar cuáles son las características o atributos que tienen los docentes que forman parte de las escuelas eficaces, pues esto permitiría disponer de evidencia que contribuya a la definición de medidas para la mejora de la calidad educativa. 


\subsection{Factores que influyen en el desempeño docente}

Existe un alto grado de acuerdo respecto de que la eficacia escolar se debe a una integración efectiva de los diferentes elementos o factores que conforman a un centro escolar, en los cuatro niveles en que éste puede ser estudiado (Murillo, 2007); el trabajo del profesor incide, sin duda, en cada uno de ellos, a la vez que también es afectado por los mismos.

En el Modelo Iberoamericano de Eficacia Escolar, entre los factores donde más influye el profesor están, por ejemplo, en el nivel alumno: las expectativas que el profesor mantiene hacia cada estudiante; en el nivel escuela: las características de los docentes, misión de la escuela (en cuanto a debate pedagógico), el compromiso de los docentes y el trabajo en equipo, el clima escolar, las expectativas y el desarrollo profesional de los docentes; y los ya mencionados en el nivel aula.

Scheerens (2016), por su parte, establece que el estudio del desempeño docente debe ser diferenciado en función de dos variables: la enseñanza eficaz y la eficacia docente. La primera está referida a los procesos de enseñanza, y está compuesta por seis variables globales: curricular; gestión del salón de clases realizada por el profesor; estrategia de enseñanza estructurada; estrategia de enseñanza orientada de manera constructiva; clima, apoyo e interacciones positivas; y evaluación y monitoreo. La eficacia docente se enfoca en las características del profesor: rasgos de personalidad; calificaciones formales y experiencia; pericia en la materia y conocimiento sobre enseñanza y aprendizaje; y conocimiento pedagógico del contenido. El análisis de ambas variables generales permitiría obtener una noción más completa de los factores que afectan al desempeño docente.

\subsection{Modelos para la enseñanza eficaz}

El interés por mejorar la calidad de la educación ha llevado a diferentes investigadores a estudiar cuáles son los factores que afectan la enseñanza que llevan a cabo los docentes. Los hallazgos han permitido clasificarlos en internos o externos, donde los primeros son aquellos inherentes al profesor o que dependen de su voluntad y rasgos de personalidad; y los segundos dependen de aspectos ajenos a él, más propios de las condiciones del contexto escolar o de la escuela misma.

Entre los investigadores contemporáneos que se han enfocado en esta temática y cuyos trabajos han destacado en el plano internacional por sus aportaciones están Danielson (2013), quien planteó el Marco para la enseñanza, y Stronge (2018), con su propuesta de Cualidades de los maestros eficaces. Por otra parte, en México se han desarrollado propuestas encaminadas a la evaluación del desempeño docente, donde se integran los factores propios de la práctica docente de calidad; como ejemplo se tiene el Perfil, parámetros e indicadores para docentes y técnicos docentes en Educación Media Superior (PPI), desarrollado por la SEP (2018), a partir del modelo de Danielson. A manera de síntesis, se presenta en el cuadro 1 los modelos para el desempeño docente, propuestos por los autores mencionados y por la SEP, para fines de comparación.

Se identificó que los tres modelos incluyeron la formación y desarrollo docentes, así como la planeación didáctica, lo que permite inferir que estos factores son esenciales dentro de la práctica docente (cuadro 1). Como referente para esta investigación, se optó por el modelo planteado por Stronge, debido a la robustez de sus fundamentos teóricos y a que 
los componentes específicos de su propuesta permiten un mejor acercamiento y conocimiento de los aspectos vinculados con la práctica docente.

Cuadro 1. Modelos propuestos para el desempeño docente: Danielson (2013), Stronge (2018) y SEP (2018)

\begin{tabular}{|c|c|c|c|}
\hline DIMENSIONES & STRONGE & DANIELSON & SEP \\
\hline $\begin{array}{c}\text { Formación y } \\
\text { desarrollo } \\
\text { docentes }\end{array}$ & $\begin{array}{l}\text { Prerrequisitos para una } \\
\text { enseñanza efectiva }\end{array}$ & $\begin{array}{l}\text { Responsabilidades } \\
\text { profesionales }\end{array}$ & $\begin{array}{l}\text { Desarrollo propio en la } \\
\text { función } \\
\text { Conocimientos para el } \\
\text { desempeño de la } \\
\text { función docente }\end{array}$ \\
\hline $\begin{array}{c}\text { Disposición } \\
\text { hacia los } \\
\text { alumnos } \\
\end{array}$ & $\begin{array}{l}\text { El profesor como } \\
\text { persona }\end{array}$ & - & - \\
\hline Gestión del aula & $\begin{array}{l}\text { Gestión y organización } \\
\text { del aula }\end{array}$ & El entorno del aula & - \\
\hline $\begin{array}{l}\text { Planeación } \\
\text { didáctica }\end{array}$ & $\begin{array}{l}\text { Planificación y } \\
\text { organización de la } \\
\text { instrucción }\end{array}$ & $\begin{array}{l}\text { Planificación y } \\
\text { preparación }\end{array}$ & Práctica docente \\
\hline Instrucción & $\begin{array}{l}\text { Implementación de la } \\
\text { instrucción }\end{array}$ & Instrucción & - \\
\hline $\begin{array}{l}\text { Monitoreo del } \\
\text { aprendizaje }\end{array}$ & $\begin{array}{l}\text { Monitoreo del progreso } \\
\text { y potencial del } \\
\text { estudiante }\end{array}$ & - & - \\
\hline $\begin{array}{l}\text { Vinculación con } \\
\text { el entorno }\end{array}$ & 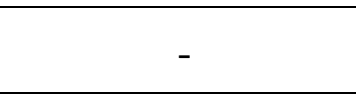 & - & $\begin{array}{l}\text { Vínculo con el contexto } \\
\text { escolar }\end{array}$ \\
\hline $\begin{array}{l}\text { Ética y } \\
\text { compromiso }\end{array}$ & - & - & $\begin{array}{l}\text { Normativa y ética en la } \\
\text { función }\end{array}$ \\
\hline
\end{tabular}

\section{Método}

Esta investigación se realizó con base en un método mixto, y un diseño secuencial explicativo (Creswell, 2014). Este tipo de acercamientos favorecen una mayor comprensión del objeto de estudio debido a que, como lo explica Arroyo (2009) permiten, en primer lugar, obtener dos imágenes distintas de una misma realidad (complementación); en segundo lugar, ofrece resultados de mayor calidad al subsanar las carencias de un solo acercamiento (combinación), y en tercer lugar, verifica la consistencia de los resultados tanto en un acercamiento como en el otro (triangulación). Se realizaron dos acercamientos. En el primero, de carácter cuantitativo, se estableció el nivel de eficacia escolar de las instituciones de EMS escolarizadas del estado de Aguascalientes (México), a través de modelos jerárquicos lineales (HLM por sus siglas en inglés). Y el segundo, que es el foco de este documento, fue con base en un acercamiento de tipo cualitativo, empleando entrevistas semiestructuradas dirigidas a directivos de los centros escolares. Se describen a continuación.

\subsection{Análisis cuantitativo para la selección de las escuelas}

Para esta fase del estudio, se seleccionaron 23 escuelas consideradas de baja eficacia y 19 de alta eficacia, correspondientes a la población conformada por 119 centros de la 
modalidad escolarizada pertenecientes a los diferentes subsistemas de enseñanza en el estado.

Se utilizaron las puntuaciones obtenidas por los estudiantes de sexto semestre de centros de EMS (Educación Secundaria, $2^{\circ}$ nivel) en el Examen Nacional de Ingreso a la Educación Superior (EXANI II), considerando seis generaciones, de 2012 a 2017. Esta prueba se aplica junto con un cuestionario de contexto de manera censal en el estado de Aguascalientes, México (Centro Nacional de Evaluación para la Educación Superior [CENEVAL], 2017). El cuadro 2 muestra el total de jóvenes y escuelas que fueron evaluadas al egreso de la educación media superior del 2012 al 2017.

Cuadro 2. Estudiantes y escuelas de evaluados en cada ciclo escolar en el estado de Aguascalientes

\begin{tabular}{lcccccc}
\hline & $\mathbf{2 0 1 2}$ & $\mathbf{2 0 1 3}$ & $\mathbf{2 0 1 4}$ & $\mathbf{2 0 1 5}$ & $\mathbf{2 0 1 6}$ & $\mathbf{2 0 1 7}$ \\
\hline Estudiantes & 10.507 & 10.616 & 10.904 & 11.120 & 12.073 & 12.407 \\
Escuelas & 145 & 151 & 156 & 168 & 173 & 184 \\
\hline
\end{tabular}

Fuente: Elaboración propia.

Fue necesario considerar instituciones en las que participaran más de 10 alumnos en la aplicación de la prueba mencionada, y que estuvieran en las seis mediciones, siendo un total de 119 centros. A continuación, se enlistan las variables que se emplearon para el desarrollo de los modelos:

\section{Variable dependiente}

- Índice CENEVAL: promedio de las puntuaciones en las cinco áreas que mide el examen; es determinado por el CENEVAL.

\section{Nivel 1. Covariables del nivel del estudiante}

- Índice socioeconómico y cultural (ISEC): construido a partir de componentes: escolaridad madre, escolaridad padre, cantidad de libros en casa; y los siguientes bienes y servicios: telefonía fija, lavadora, refrigerador, horno de microondas, Internet, televisión de paga, reproductor de video, computadora, televisión, número de automóviles y número de baños completos.

- Sexo.

- Promedio bachillerato.

- Trabajo remunerado en horas.

- Expectativa de estudios: máximo nivel de estudios que le gustaría alcanzar (técnico, licenciatura o posgrado).

- Extra-edad: edad normativa $=0$; edad mayor a la normativa $=1$

Nivel 2. Covariables del nivel de escuela

- N2_Sexo: proporción de mujeres en la escuela.

- Sostenimiento privado: Público $=0 ;$ Privado $=1$.

- N2_promedio bachillerato (a nivel de la escuela).

- Tamaño de la escuela: número de alumnos que egresan en el sexto semestre.

- N2_ ISEC (promedio de la escuela). 
Se desarrolló tanto un análisis transversal contextualizado (OCDE, 2011) como un análisis del cambio temporal a nivel de escuelas. El primero fue un diseño multinivel en el que los datos se agrupan de forma anidada, que de acuerdo con Snijders (2014) implica más de una unidad de análisis y explora los efectos que las distintas variables tienen en cada uno de los niveles involucrados. Para los análisis transversales contextualizados fueron necesarias las variables a nivel de los estudiantes (nivel 1), y de las escuelas (nivel 2). Respecto del estudio del cambio en el tiempo, se realizó al nivel de las escuelas pues las evaluaciones EXANI II son cortes transversales con base en las cohortes correspondientes al egreso de la EMS. Los análisis fueron realizados con base en la siguiente expresión:

$$
\text { Nivel } 1 \text { (estudiantes) Nivel } 2 \text { (centros) }
$$

Parte estructural

$$
Y_{i j}=\beta_{0 j}+\sum_{q=1}^{Q} \beta_{q j} X_{q i j}+r_{i j}
$$

Parte probabilística

$$
r_{i j} \sim N\left(0, \sigma^{2}\right)
$$

$$
\beta_{0 j}=\gamma_{00}+\sum_{s=1}^{S} \gamma_{0 s} W_{s j}+u_{0 j}
$$$$
u_{0 j} \sim N\left(0, \tau_{00}\right)
$$

Una escuela fue considerada de alta eficacia si presentaba una pendiente grande y positiva; por el contrario, escuelas con pendiente grande pero negativa, fueron identificadas como de baja eficacia.

Una vez efectuados los análisis estadísticos, se definieron cuatro criterios para seleccionar a las escuelas, los que se describen a continuación:

- Residuos Extremos (RE). A partir del promedio de los residuos de los análisis transversales contextualizados de los seis años, se selecciona a las escuelas de mayor y menor residuo.

- Crecimientos de los Residuos (CR). Se calcula una función lineal entre los promedios de los residuos de las escuelas en los seis años, se selecciona a las pendientes de mayor y menor crecimiento.

- Puntuaciones Brutas Extremas (PE). Se promedian las puntuaciones brutas de la variable dependiente y se selecciona a las de mayor y menor puntuación.

- Crecimiento de las Puntuaciones Brutas (cP). Se calcula una función lineal entre los promedios de las puntuaciones brutas de las escuelas en los seis años y se selecciona a las pendientes de mayor y menor crecimiento.

Para mayor comprensión del procedimiento estadístico desarrollado para selección de las escuelas, se puede consultar el artículo de Pedroza, Peniche y Lizasoain (2018), en donde se describe a detalle los modelos realizados y los argumentos de cada momento en el proceso. Este tipo de análisis ha sido empleado de manera tradicional en el enfoque de escuelas eficaces, presentándose en trabajos como los de Castro y Lizasoain (2012) y Lizasoain y Angulo (2014).

\subsection{Análisis cualitativo para el acercamiento con directores}

Una vez seleccionadas las escuelas se dio inicio al segundo acercamiento, de carácter cualitativo que es el foco de este artículo. 
Para esta fase del estudio, se contactó a cada directivo y se solicitó su participación en el estudio; al final, aceptaron participar 17 planteles de alta eficacia y 21 de baja, haciendo un total de 38 entrevistas realizadas a directores escolares.

Este acercamiento al ser de tipo cualitativo, y en consistencia con las aportaciones de Stake (2007) se centró en la descripción y, principalmente, en la búsqueda de la comprensión acerca de la forma en la que el desempeño docente es influido por las categorías que componen el modelo de eficacia escolar, centrando la atención en casos específicos más allá de buscar generalizaciones hacia toda la EMS. El trabajo se abordó desde la perspectiva de los directores de cada plantel y el procedimiento metodológico consistió en:

- Revisión de la literatura y entendimiento del modelo teórico;

- Estudio y selección cuidadosa de los informantes;

- Diseño de los instrumentos y establecimiento de los protocolos, y

- Desarrollo de las entrevistas y análisis de datos.

Respecto de los instrumentos, se diseñó y empleó una cédula de datos generales tanto del plantel como de su directivo; en esta cédula se consideraron datos como, edad del directivo, formación profesional, experiencia en años como directivo escolar, cursos de formación continua y datos sobre la matrícula de la escuela.

En cuanto a la guía de entrevista semi-estructurada, se establecieron ejes de exploración con base en los factores del modelo de la eficacia escolar así como en los modelos de desempeño docente (ya descritos), especialmente el aportado por Stronge (2018) a través de preguntas sobre los logros escolares, el clima, las estrategias de enseñanza que emplean los profesores, el contacto con la comunidad, así como procesos para atender a los grupos vulnerables.

Cada entrevista fue audio-grabada, previo consentimiento informado. Las entrevistas fueron transcritas, descompuestas en pequeñas unidades de análisis o categorías para su posterior análisis con base en las diferentes dimensiones del modelo de la eficacia las cuales fueron seleccionadas para fines del estudio. Para ello, se procedió a clasificar temáticamente las ideas expresadas por los entrevistados, empleando un sistema de categorización considerando ideas homogéneas que permitieron entendimientos generales lo que caracteriza al método inductivo.

Se realizó un análisis de contenido a las respuestas de los participantes, que permitió desarrollar interpretaciones a la luz de la teoría lo que derivó en las conclusiones que se presentan para responder a los objetivos del presente estudio. El resultado del grupo de categorías, por tipo de factor, se muestra en el cuadro 3.

Cuadro 3. Factores y categorías para el análisis de datos

\begin{tabular}{ll}
\hline \multicolumn{1}{c}{ FACTORES } & \multicolumn{1}{c}{ CATEGORÍAS } \\
\hline Internos & Compromiso docente \\
\hline & Clima organizacional \\
& Dotación de materiales y recursos para la enseñanza \\
Externos & Capacitación y actualización \\
& Estabilidad laboral \\
& Carga horaria \\
\hline
\end{tabular}

Fuente: Elaboración propia. 
A partir de la definición de los factores y las categorías mencionados, se seleccionaron expresiones exactas de los participantes para evidenciar el conjunto de hallazgos que a continuación de reporta.

\section{Resultados}

La información obtenida de las entrevistas realizadas a los directivos de las escuelas participantes, muestra la existencia de aspectos que afectan el desempeño docente; en algunos casos son coincidentes tanto en escuelas de alta como de baja eficacia, y en otros se encuentran diferencias de manera notable. Los factores relativos al desempeño docente se clasificaron en dos grupos: internos y externos. El primero de ellos corresponde a cuestiones propias del docente, en donde su implicación es directa: compromiso docente. Las categorías incluidas en la agrupación de factores externos, son aquellas que afectan al docente, sin que él tenga una injerencia directa, son elementos o circunstancias dadas por el entorno (la escuela o el sistema educativo): clima organizacional, dotación de materiales y recursos para la enseñanza, capacitación y actualización, estabilidad laboral y carga horaria.

En el cuadro 4 se presenta una síntesis de los resultados obtenidos, donde la X representa la presencia de las categorías, identificadas a partir de las respuestas emitidas por los directores; la presencia de tales categorías es explicada en apartados posteriores con fragmentos de las reflexiones emitidas por los directivos escolares.

Cuadro 4. Factores y categorías que afectan el desempeño docente de escuelas de alta y baja eficacia

\begin{tabular}{llcc}
\hline \multirow{2}{*}{ FACTORES } & \multirow{2}{*}{ CATEGORías } & \multicolumn{2}{c}{ EFICACIA } \\
\cline { 3 - 4 } & & Baja & Alta \\
\hline Internos & Compromiso docente & $\mathrm{X}$ & $\mathrm{X}$ \\
\hline \multirow{5}{*}{ Externos } & Clima organizacional & $\mathrm{X}$ & $\mathrm{X}$ \\
& Dotación de materiales y recursos para la enseñanza & $\mathrm{X}^{*}$ & $\mathrm{X}$ \\
& Capacitación y actualización & & $\mathrm{X}$ \\
& Estabilidad laboral & & $\mathrm{X}$ \\
\hline
\end{tabular}

Nota: * Existe presencia del factor, sin embargo, el cumplimiento no se da en su totalidad.

Fuente: Elaboración propia.

En cuanto al grupo de factores internos, el compromiso docente presentó una diferencia entre ambos tipos de escuelas. En el conjunto de factores externos la estabilidad laboral y la carga horaria parecen marcar diferencias entre las escuelas de alta y baja eficacia.

En los apartados que continúan, se presentan los resultados específicos para los factores tanto internos como externos, enlistados en la tabla 3. Cuando se identificaron contrastes en las categorías de los factores por tipo de eficacia de las escuelas, se optó por presentar las evidencias en forma de tabla, con la intención de facilitar la comparación.

\subsection{Factores internos}

Referente al factor de Compromiso docente, las diferencias entre ambos tipos de escuela son evidentes. Los directores de los centros escolares de alta eficacia expresaron que el compromiso de los profesores se manifiesta en el interés que estos demuestran por utilizar e incluso generar materiales didácticos pertinentes a las necesidades de los estudiantes; un 
marcado interés por optimizar el tiempo disponible a lo largo de la jornada escolar, de tal forma que sea invertido en mayor medida en actividades enfocadas en el aprendizaje; y una disposición para aceptar e implementar los cambios normativos establecidos para este nivel educativo.

En el cuadro 5 se incluyen los testimonios de los directivos para cada una de las categorías identificadas, donde se evidencia el compromiso de los docentes, según el tipo de eficacia del centro escolar. Vale la pena comentar, que los espacios vacíos referidos a los centros de baja eficacia, se debió a que no se encontró información referida a las subcategorías.

Cuadro 5. Subcategorías identificadas en la categoría Compromiso docente, según el tipo de eficacia

\begin{tabular}{|c|c|c|}
\hline \multirow{2}{*}{ SUBCATEGORÍAS } & \multicolumn{2}{|c|}{ TIPO DE EFICACIA } \\
\hline & Baja & Alta \\
\hline $\begin{array}{l}\text { Desarrollo de } \\
\text { materiales propios }\end{array}$ & & $\begin{array}{l}\text { En matemáticas el libro de texto es } \\
\text { generado por los propios profes, no } \\
\text { por material externo. (07-A- } \\
\text { O6SUB) }\end{array}$ \\
\hline $\begin{array}{l}\text { Disposición para } \\
\text { asistir a reuniones } \\
\text { en horarios que no } \\
\text { afectan las clases }\end{array}$ & & $\begin{array}{l}\text { [Las reuniones docentes] se realizan } \\
\text { a partir de las 11:00 de la mañana } \\
\text { para no dejar de lado las clases y es } \\
\text { iniciativa de los docentes. (03-A- } \\
\text { 05D) }\end{array}$ \\
\hline $\begin{array}{l}\text { Adaptación a los } \\
\text { cambios } \\
\text { normativos }\end{array}$ & & $\begin{array}{l}\text { [Hay] situaciones de cambio en la } \\
\text { cual todos nos tenemos que adaptar } \\
\text { [...] y poder prestar el servicio con } \\
\text { la mejor calidad que podamos a } \\
\text { pesar de los pesares. (o2-A-10D) }\end{array}$ \\
\hline Autonomía & $\begin{array}{l}\text { Pues cada docente tiene su forma } \\
\text { particular de dar sus clases } \\
\text { algunos [...] dependiendo del } \\
\text { tema que se vaya a emplear decide } \\
\text { qué recursos utilizar. (04-B- } \\
\text { o2D) }\end{array}$ & $\begin{array}{l}\text { Es muy diverso porque al margen de } \\
\text { los programas que son los mismos, } \\
\text { hay libertad de cátedra, entonces, } \\
\text { mientras cumpla con los programas } \\
\text { es un poco libre. (O7-A-O6SUB) }\end{array}$ \\
\hline
\end{tabular}

Fuente: Elaboración propia.

De acuerdo con la información expresada por los directores, existen claras diferencias entre las tres primeras categorías referidas al compromiso docente, sin embargo, coinciden en cuanto a que los profesores ejercen la autonomía en el desarrollo de sus clases, al decidir la manera en que implementan sus programas de curso.

\subsection{Factores externos}

Al igual que en el primer factor, en esta sección se describen los factores externos, considerando que los espacios vacíos de las tablas manifiestan una ausencia de expresiones respecto de las subcategorías citadas. En relación a la percepción del clima organizacional, los directores reconocen que las relaciones humanas son algo complicado; a pesar de ello, refirieron que, de manera general, el clima en las escuelas es bueno, de cordialidad y respeto.

Muy cordial, claro que hay diferencias con algunos maestros es normal en toda actividad humana ¿iverdad?, [...] pero en general la visión, sí es bueno. (01-A$11 \mathrm{DEC})$ 
En cuanto a la categoría Dotación de materiales y recursos para la enseñanza, en ambos tipos de escuela los directores manifiestan que se provee a los profesores los recursos didácticos que requieren para su práctica docente. De esta manera, con estas acciones, reflejan que desde el plano administrativo es importante que los profesores cuenten con los materiales didácticos, recursos tecnológicos y espacios necesarios para realizar su función bajo la perspectiva de que esto influye en un mejor desempeño.

Dentro de los factores externos también se exploró la Capacitación y actualización, que se compone por dos subcategorías como se muestra en la tabla 6: participación docente en cursos por iniciativa propia, y participación docente en cursos que ofrece la escuela. Las instituciones de alta eficacia se caracterizaron por tener una participación más activa por parte de los docentes en ambos tipos de oferta, así como por facilitarles un mayor número de experiencias de aprendizaje.

Cuadro 6. Categorías y subcategorías que promueven el trabajo docente en bachilleratos de alta y baja eficacia

\begin{tabular}{|c|c|c|c|}
\hline \multirow{2}{*}{ CATEGORÍAS } & \multirow{2}{*}{ SUBCATEGORÍAS } & \multicolumn{2}{|c|}{ TIPO DE EFICACIA } \\
\hline & & Baja & Alta \\
\hline \multirow{2}{*}{$\begin{array}{l}\text { Capacitación y } \\
\text { actualización }\end{array}$} & $\begin{array}{l}\text { Participación } \\
\text { docente en } \\
\text { cursos por } \\
\text { iniciativa } \\
\text { propia }\end{array}$ & $\begin{array}{l}\text { Tenemos una plantilla } \\
\text { docente que se } \\
\text { preocupa por estar al } \\
\text { dia, por estar } \\
\text { actualizado y } \\
\text { empatarse al servicio } \\
\text { profesional. (o2-B- } \\
\text { o1D) }\end{array}$ & $\begin{array}{l}\text { Hay una cantidad considerable, } \\
\text { [...] de maestros que entraron a } \\
\text { los diplomados. (o9-A-06SUB) }\end{array}$ \\
\hline & $\begin{array}{l}\text { Participación } \\
\text { docente en } \\
\text { cursos que } \\
\text { ofrece la } \\
\text { escuela }\end{array}$ & & $\begin{array}{l}\text { Cada mes tenemos ya sea } \\
\text { conferencia, un curso [...] Si } \\
\text { hay oportunidad de algún buen } \\
\text { curso en donde algunos maestros } \\
\text { puedan participar [la escuela] } \\
\text { aporta parte o el pago completo } \\
\text { de este. (08-A-04SUB) }\end{array}$ \\
\hline $\begin{array}{l}\text { Estabilidad } \\
\text { laboral }\end{array}$ & $\begin{array}{l}\text { Continuidad } \\
\text { laboral en la } \\
\text { misma escuela }\end{array}$ & & $\begin{array}{l}\text { Trato de evitar no tener a un } \\
\text { maestro en un semestre y a otro } \\
\text { semestre tener otro [...] estoy } \\
\text { cien por ciento segura de que esa } \\
\text { es una variable [la estabilidad } \\
\text { laboral] es bien importante para } \\
\text { obtener resultados. (o2-A-10D) }\end{array}$ \\
\hline Carga horaria & $\begin{array}{l}\text { Tiempo } \\
\text { completo o } \\
\text { medio tiempo }\end{array}$ & & $\begin{array}{l}\text { Tenemos mucho personal de } \\
\text { tiempo completo, yo creo que eso } \\
\text { es bueno para la institución } \\
\text { porque genera un compromiso } \\
\text { del docente. (03-A-05D) }\end{array}$ \\
\hline
\end{tabular}

Fuente: Elaboración propia.

En continuidad con los factores externos, la estabilidad laboral fue identificada como una categoría que incide en el desempeño de los docentes de las escuelas de alta eficacia, permitiendo que el profesor se mantenga laborando en la misma escuela. Con base en los testimonios, se interpreta que los centros escolares están sujetos a una alta tasa de movilidad por parte del profesorado, lo que podría afectar al logro de los objetivos educativos. ¿Cómo se puede explicar esta situación? Una respuesta puede ser que los 
docentes suelen trabajar en diversas escuelas ante la falta de un contrato de tiempo completo en una escuela, o que las condiciones laborales no son las esperadas en comparación con las otorgadas en otros empleos, lo que puede afectar su disponibilidad para participar de los proyectos escolares.

En cuanto a la categoría carga horaria, que consiste en la cantidad de horas que el profesor tiene asignadas para sus labores en un centro escolar, este incluyó la subcategoría tiempo completo o medio tiempo, y fue mencionada por los directivos de escuelas de alta eficacia. El comentario identificado alude a que es bueno para la institución el disponer de personal que tenga dedicación de tiempo completo para desempeñar sus labores, porque incrementa el compromiso de los docentes ante los objetivos institucionales.

Las entrevistas permitieron conocer la visión del directivo respecto de la importancia que tienen los docentes para el logro de los objetivos institucionales, como bien se expresa:

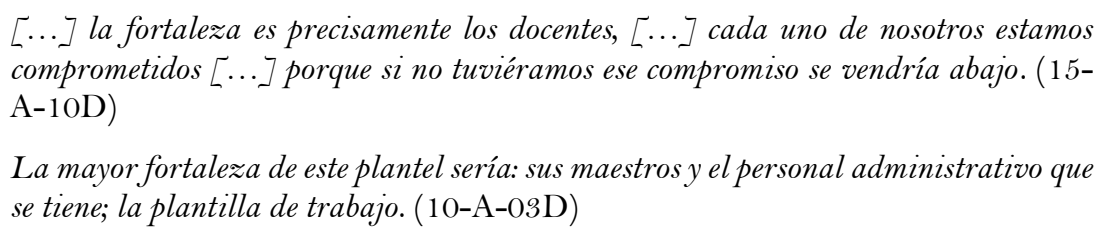

Dejaron de manifiesto que existen un conjunto de categorías ajenas a la intervención de los profesores que inciden de manera importante en su función, sin embargo, también expusieron la relevancia que tienen categorías como el compromiso docente para mejorar el logro de los estudiantes.

\section{Discusión y conclusiones}

La opinión del directivo escolar es una fuente de gran riqueza que vale la pena explorar; con base en ello, se pudo determinar que el compromiso docente es, en definitiva, un factor clave para el éxito de la escuela y de la educación, pues es gracias a él que los docentes se muestran con la disposición incluso de diseñar materiales especiales, o bien de asistir a reuniones fuera del horario laboral, con el interés de optimizar el tiempo en el que se encuentran los estudiantes en la escuela, así lo reconocieron los directivos en consistencia con autores como Murillo (2007). Además, la eficacia es explicada también a partir del compromiso de los docentes hacia sus alumnos, su trabajo y su mejora continua (Day et al., 2009; Horn y Murillo, 2016; y Selamat y Nordin, 2014).

En cuanto al clima escolar, para Murillo (2007), el modelo latinoamericano de eficacia escolar incluye a este factor tanto en el nivel de aula como en el de escuela; en este último son importantes aspectos como la existencia de buenas relaciones entre los integrantes de la comunidad escolar y la satisfacción de los maestros. En la investigación realizada por Etxeberria, Intxausti y Azpillaga (2017), además del primer aspecto mencionado por Murillo (2007), encontraron que las buenas condiciones académicas que faciliten el aprendizaje, el consenso respecto de la visión escolar, el sentimiento de pertenencia y una administración participativa son elementos que favorecen el logro de un buen clima organizacional.

Aunque a partir del discurso de los directivos no se manifiesta diferencia en la percepción del clima en los dos tipos de escuela, es conveniente tener presente lo expresado por Scheerens (2016) acerca de que las buenas relaciones y la satisfacción deben ser consideradas como instrumentos para mejorar la eficacia y no como fines en sí mismas. 
Las respuestas de los directivos permitieron identificar que, en ambos tipos de escuelas, en cuanto a la Dotación de materiales y recursos para la enseñanza como categoría para la eficacia escolar, se da de igual manera. En relación con este aspecto, es importante tomar en cuenta lo expresado por Martínez-Garrido y Murillo (2016) en cuanto a que la diferencia en la eficacia escolar no depende necesariamente de la disponibilidad de los recursos, sino de "la utilización de recursos didácticos variados por el docente, tanto tradicionales como tecnológicos, mejora el desarrollo cognitivo de los estudiantes" (p. 494). Esto significa un aspecto más por estudiar, pues más que la presencia o ausencia de recursos, es fundamental investigar el uso que los docentes le dan a todo aquello de lo que disponen para la enseñanza y el aprendizaje, o sea, las adecuaciones que los profesores realizan, lo que depende sobremanera del compromiso, proactividad y del trabajo en equipo de los actores escolares (Figueroa, 2017).

Como sucede con otros factores que afectan el desempeño docente, la mera participación en actividades de capacitación y actualización no es suficiente para mejorar el desempeño; éstas deben proveer aprendizaje colectivo, intercambio de ideas entre profesores y colaboración para mejorar la práctica en el aula (Schleicher, 2011). Aunado a lo anterior, Santibáñez, Rubio y Vázquez (2017) expresan que a pesar de que México es uno de los países donde los profesores reciben más formación continua, este país no cuenta con mecanismos que permitan valorar la utilidad y el impacto de dicha formación, aseveración manifestada también por la Comisión para la Educación de Calidad para Todos (2016).

En relación con la capacitación y la actualización, sus resultados en el aula dependen en gran medida de que los profesores estén motivados y que se responsabilicen de su propia formación. Esto podría lograrse mediante diagnósticos realizados por la propia comunidad escolar, por ser esta la que cuenta con la experiencia de lo que acontece en los centros escolares, así como sus debilidades. A partir de ahí podrían generarse mecanismos de retroalimentación que atiendan la heterogeneidad de la EMS de forma adecuada, y así proponer estrategias de formación continua desarrolladas con el apoyo de personas altamente calificadas (Comisión para la Educación de Calidad para Todos, 2016; Rueda y Sánchez, 2018; Santibáñez, Rubio, y Vázquez, 2017).

En cuanto a la falta de estabilidad laboral que prevalece en los docentes de los centros escolares participantes, la literatura refiere una serie de problemas que pueden derivarse como son: la falta de pertenencia a un proyecto institucional, dificultad para conformar equipos de trabajo entre colegas, limitaciones en el conocimiento del alumnado más allá del aula y la limitación para establecer diálogos con los padres de familia (Observatorio Argentinos por la Educación, 2018). Por ello es fundamental que en las instituciones educativas existan políticas que aseguren que los profesores laboren en ambientes que faciliten su éxito y que motiven a los maestros efectivos a continuar en la enseñanza (Schleicher, 2011).

La comparación entre países realizada por Bruns y Luque (2014), enfocada en cómo mejorar el aprendizaje en América Latina y el Caribe indica que, si se quiere elevar la calidad docente, además de darles seguridad laboral a los profesores es prioritaria la alineación de tres tipos de incentivos: las recompensas profesionales, las presiones por rendir cuentas y las recompensas financieras, combinados de una manera dependiente en gran medida del contexto.

La carga horaria parece afectar a ambos tipos de escuelas en cuanto a la dificultad para asignar a sus docentes un contrato de tiempo completo, este como uno de los factores más 
críticos para el éxito de la educación y de la escuela (Selamat y Nordin, 2014). Por otra parte, los docentes que tienen contratos menos estables (o parciales) tienden a mostrar un menor compromiso con la institución, no se desempeñan de la manera en que lo hacen sus pares contratados de tiempo completo y generalmente faltan más en comparación con los segundos, como lo afirman Coluccio, Muñoz y Ferrer (2016). Además, según reportan López y colaboradores (2016), en el caso de México, los profesores de tiempo parcial tienen habilidades profesionales comparables a los de tiempo completo, pero las condiciones de trato institucional que reciben (como el bajo salario) limitan su desempeño profesional. Este fenómeno relacionado al tipo de contratación y tiempo de labor del docente es poco estudiado en México, y menos en la EMS, lo que significa un campo fértil para la investigación educativa.

Los hallazgos presentados son consistentes con estudios previos en el sentido de que resalta el rol del docente ante el aprendizaje de los estudiantes. A partir de la voz de los directivos de escuelas de alta y baja eficacia, abona información que manifiesta lo fundamental que es el compromiso docente, pues a partir de él es posible generar una serie de estrategias para atender a las necesidades de los estudiantes, lo que se vuelve especialmente relevante en el contexto de la EMS en México, marcado por una gran desigualdad y diversidad en cuanto a la oferta educativa. Sin embargo, este compromiso no sólo depende del docente, sino que es alimentado por una serie de factores como la estabilidad laboral y la carga horaria, gracias a los cuales este actor puede dedicar mayor energía a atender a sus alumnos.

Respecto de los modelos de desempeño docente mencionados al inicio del escrito, parece que el modelo propuesto por Stronge responde de forma más cercana a lo que se reportó en los hallazgos correspondientes a la fase cualitativa de este estudio, especialmente en lo que se refiere a los factores internos al docente como el compromiso, que puede manifestarse mediante distintas actividades enfocadas en el aprendizaje de los estudiantes, lo que podría significar ser una guía de verificación objetiva de las prácticas docentes con técnicas más profundas y directas. Conviene mencionar que el emplear la opinión del director escolar significó una exploración a lo que acontece dentro de las aulas desde la perspectiva de este actor educativo, por lo que se reconoce la necesidad de enriquecer el estudio mediante técnicas y métodos más amplios y directos al docente, como puede ser la observación del trabajo del profesor dentro del aula. Así mismo, sería fundamental escuchar la perspectiva de los profesores a fin de conocer sus prácticas, pero también sus sentimientos acerca de la labor que tienen sobre sus hombros.

Es importante mencionar que la comparación realizada en este escrito entre las escuelas por su tipo de eficacia escolar y determinado por la opinión de los directivos, no evidenció cantidad ni diversidad de prácticas en los centros de baja eficacia, esto debido a que los hallazgos fueron basados en las expresiones de los actores entrevistados; valdría la pena considerar el desarrollo de posibles estudios que amplíen la exploración al respecto.

Finalmente, este trabajo abre la puerta a estudios más profundos que permitan explorar qué es lo que ocurre al interior de las escuelas eficaces y no eficaces en este nivel educativo, así como también el reconocimiento de las buenas prácticas, de tal forma que sea posible identificar información significativa para la generación de propuestas de mejora. 


\section{Referencias}

Arroyo, M. (2009). Cualitativo-cuantitativo: La integración de las dos perspectivas. En A. Merlino (Coord.), Investigación cualitativa en ciencias sociales (pp. 56-79). Buenos Aires: CENGAGE Learning.

Backhoff, E., Guevara, G., Hernández, J. y Sánchez, A. (2018). El aprendizaje al término de la educación media superior en México. El Cotidiano Revista de la Realidad Mexicana Actual, 208(33), 7-19.

Bruns, B. y Luque, J. (2014). Profesores excelentes. Cómo mejorar el aprendizaje en América Latina y el Caribe. Washington, DC: Grupo del Banco Mundial.

Castro, M. y Lizasoain, L. (2012). Las técnicas de modelización estadística en la investigación educativa: minería de datos, modelos de ecuaciones estructurales y modelos jerárquicos lineales. Revista Española de Pedagogía 70(251), 131-148.

Centro Nacional de Evaluación para la Educación Superior. (2017). Exámenes nacionales de ingreso, EXANI II. Recuperado de http://www.ceneval.edu.mx/exani-ii

Chapman, C., Reynolds, D., Muijs, D., Sammons, P., Stringfield, S. y Teddlie, C. (2016). Educational effectiveness and improvement research and practice: The emergence of the disicpline. En C. Chapman, D. Muijs, D. Reynolds, P. Sammons y C. Teddlie, The Routledge international handbook of educational effectiveness and improvement. Research, policy, and practice (pp. 3-24). Nueva York, NY: Routledge. https://doi.org/10.4324/9781315679488

Coluccio, A., Muñoz, C. y Ferrer, R. (2016). Situación contractual y su relación con satisfacción laboral, clima organizacional y absentismo en docentes. Salud y Sociedad, 7(1), 98-111. https://doi.org/10.22199/S07187475.2016.0001.00006

Comisión para la Educación de Calidad para Todos. (2016). Construyendo una educación de calidad: Un pacto con el futuro de América Latina. Buenos Aires: Santillana.

Creemers, B. y Kyriakides, L. (2008). The dynamics of educational effectiveness: A contribution to policy practice and theory in contemporary schools. Londres: Routledge. https://doi.org/10.4324/9780203939185

Creswell, J. W. (2014). Research design: Qualitative, quantitative, and mixed methods approaches. Londres: SAGE.

Danielson, C. (2013). The Danielson group. Recuperado de https://www.danielsongroup.org/framework/

Davis, G. y Thomas, M. (2007). Escuelas eficaces y profesores eficientes. Madrid: La Muralla.

Day, C., Sammons, P., Hopkins, D., Harris, A., Leithwood, K., Gu, Q., Brown, E, Ahtaridou, E. y Kington, A. (2009). The impact of school leadership on pupil outcomes. Final report. Londres: UK Government Department for Children, Schools and Families.

Diario Oficial de la Federación. (2012). Decreto por el que se declara reformado el párrafo primero; el inciso c) de la fracción II y la fracción $V$ del artículo 30., y la fracción I del artículo 31 de la Constitución Política de los Estados Unidos Mexicanos. Recuperado de http://www.dof.gob.mx/nota_detalle.php?codigo=5233070

Etxeberria, F., Intxausti, N. y Azpillaga, V. (2017). School climate in highly effective schools in the autonomous region of the Basque country (Spain). REICE. Revista Iberoamericana sobre Calidad, Eficacia y Cambio en Educación, 15(4), 5-26. https://doi.org/10.15366/reice2017.15.4.001 
Figueroa, V. (2017). Eficacia escolar en entornos vulnerables de la República Dominicana (Tesis doctoral). Universidad Autónoma de Madrid.

Horn, A. y Murillo, F. J. (2016). Incidencia de la dirección escolar sobre el compromiso de los docentes: Un estudio multinivel. Psicoperspectivas, 15(2), 64-77. https://doi.org/10.5027/psicoperspectivas-Vol 15-Issue2-fulltext-746

IEA. (2017). Las cifras de la educación. Educación media superior. Fin de ciclo 2015-2016 inicio de ciclo 2016-2017. Aguascalientes: Instituto de Educación de Aguascalientes.

Instituto Nacional para la Evaluación de la Educación. (2015). Los docentes en México. Informe 2015. Ciudad de México: Instituto Nacional para la Evaluación de la Educación.

Instituto Nacional para la Evaluación de la Educación. (2017). Directrices para mejorar la permanencia escolar en la educación media superior. Ciudad de México: Instituto Nacional para la Evaluación de la Educación.

Instituto Nacional para la Evaluación de la Educación. (2018). La educación obligatoria en México. Informe 2018. Ciudad de México: Instituto Nacional para la Evaluación de la Educación.

Lizasoain, L. y Angulo, A. (2014). Buenas prácticas de escuelas eficaces del País Vasco. Metodología y primeros resultados. Participación Educativa, 3(4), 17-28.

López, A., García, O., Pérez, R., Montero, V. y Rojas, E. (2016). Los profesores de tiempo parcial en las universidades públicas estatales: Una profesionalización inconclusa. Revista de la Educación Superior, 45(180), 23-39. https://doi.org/10.1016/j.resu.2016.06.007

Martínez-Garrido, C. y Murillo, F. J. (2016). Investigación iberoamericana sobre enseñanza eficaz. Revista Mexicana de Investigación Educativa, 21(69), 471-499.

Murillo, F. J. (2007). Investigación iberoamericana sobre eficacia escolar. Bogotá: Convenio Andrés Bello.

Observatorio Argentinos por la Educación. (2018). Uno de cada 3 maestros trabaja en más de una escuela. Recuperado de

https://cms.argentinosporlaeducacion.org/media/reports/Argentinos_por_la_Educacion _Infografia_02_Septiembre2018_Imprimible_1.pdf

Organización para la Cooperación y el Desarrollo Económico. (2011). La medición del aprendizaje de los alumnos: Mejores prácticas para evaluar el valor agregado de las escuelas. París: OECD Publishing.

Pedroza, H., Peniche, R. y Lizasoain, L. (2018). Criterios para la identificación y selección de escuela eficaces de nivel medio superior. Revista Electrónica de Investigación Educativa, 20(1), 14-25. https://doi.org/10.24320/redie.2018.20.1.2170

Rueda, M. y Sánchez, M. (2018). Propuesta para identificar necesidades educativas regionales, a partir de la investigación educativa. En M. Rueda y L. Álvarez, Propuestas para transformar el sistema educativo mexicano (pp. 13-16). Ciudad de México: Instituto de Investigaciones sobre la Universidad y la Educación.

Ruiz, M. M. y Luna, A. (2017). El derecho a la educación en el nivel medio superior en México. Revista Latinoamericana de Educación Inclusiva, 11(2), 73-90. https://doi.org/10.4067/S0718-73782017000200006

Santibáñez, L., Rubio, D. y Vázquez, M. (2017). Formación continua de docentes: Política actual en México y buenas prácticas nacionales e internacionales. Ciudad de México: INEE/BID.

Scheerens, J. (2016). Educational effectiveness and ineffectiveness. A critical review of the knowledge base. Nueva York, NY: Springer. https://doi.org/10.1007/978-94-017-7459-8 
Schleicher, A. (2011). Building a high-quality teaching profession: Lessons from around the world. París: OCDE Publishing. https://doi.org/10.1787/9789264113046-en

Selamat, N. y Nordin, N. (2014). Teacher's commitment and emotional intelligence: Evidence from Malaysian schools. International Journal of Science Commerce and Humanitiesm, 15, 49-59.

Secretaría de Educación Pública. (2012). Gestión estratégica en las escuelas de calidad: Orientaciones prácticas para directivos y docentes. Compendio de módulos para una gestión educativa estratégica. Ciudad de México: Secretaría de Educación Pública.

Secretaría de Educación Pública. (2018). Servicio profesional docente. Ciudad de México: Secretaría de Educación Pública.

Snijders, T. (2014). Multilevel analysis. Recuperado de http://link.springer.com.dibpxy.uaa.mx/referenceworkentry/10.1007/978-3-642-048982_387

Stake, R. E. (2007). Investigación con estudio de casos. Madrid: Morata.

Stronge, J. (2018). Qualities of effective teachers. Alexandria: Association for Supervision and Curriculum Development.

Tuirán, R. y Hernández, D. (2016). Desafíos de la educación media superior en México. Recuperado de http://www.estepais.com/articulo.php?id=460\&t=desafios-de-la-educacion-

\section{Breve CV de los autores}

\section{Rubí Surema Peniche Cetzal}

Doctora en Investigación Educativa por la Universidad de Granada, España. Licenciada en Educación y Maestra en Investigación Educativa por la Universidad Autónoma de Yucatán, México. Actualmente se desempeña como profesora investigadora en el Departamento de Educación de la Universidad Autónoma de Aguascalientes, México. Se ha desempeñado como docente en educación superior desde 2005 y ha dirigido diversas tesis a nivel pregrado y posgrado. Cuenta con un número considerable de publicaciones tanto en revistas como libros especializados a nivel nacional e internacional. Coordina el proyecto Modelo comprehensivo de la mejora de la eficacia escolar en educación media superior en Aguascalientes, México con vigencia 2018-2021. Sus intereses de investigación versan sobre la eficacia escolar, las prácticas docentes, equidad educativa, prácticas de liderazgo. ORCID ID: https://orcid.org/0000-0003-0105-6471. Email: rupeniche81@gmail.com

\section{Cristóbal Ramón Mac}

Licenciado en Educación y Doctorado en Ciencias Sociales por la Universidad Autónoma de Yucatán, y Maestría en Desarrollo Organizacional por el INESPO. Con experiencia docente en pregrado y posgrado, en las áreas de didáctica, diseño curricular, liderazgo educativo, gestión educativa, evaluación de instituciones educativas e investigación educativa. Actualmente realiza estancia posdoctoral en la Universidad Autónoma de Aguascalientes, con apoyo del CONACYT. Sus áreas de investigación se enfocan en la eficacia escolar en educación media superior, la práctica docente en escuelas eficaces y la gestión de centros escolares. ORCID ID: https://orcid.org/0000-0003-3732-5717. Email: ccrmac@gmail.com 


\section{Cintya Guzmán Ramírez}

Doctora y Maestra en Investigación Educativa por la Universidad de Aguascalientes. Licenciada en Psicología por la Benemérita Universidad Autónoma de Puebla. Actualmente Jefa de Departamento de Investigación Educativa en el Instituto de Educación de Aguascalientes, área en la que tiene a su cargo la coordinación de proyectos de investigación, así como el desarrollo de actividades de difusión de resultados en esta materia. Anteriormente colaboró en proyectos de investigación en el Departamento de Investigación Educativa de la Universidad Autónoma de Aguascalientes relacionados con la transición de los estudiantes de bachillerato hacia la educación superior, así como con la violencia escolar. ORCID ID: https://orcid.org/0000-0002-8042-5900. Email: cintyaguzr@gmail.com

\section{Noé Mora Osuna}

Licenciado en Ciencias de la Educación por la Universidad Autónoma de Baja California. Ha participado en proyectos de investigación sobre eficacia y mejora escolar, así como enseñanza eficaz. Actualmente es profesor investigador asociado por la Universidad Autónoma de Aguascalientes, asignado al Departamento de Educación. Es colaborador en el proyecto Modelo comprehensivo de la mejora de la eficacia escolar en educación media superior en Aguascalientes, México. Sus líneas de interés son eficacia escolar, enseñanza eficaz, convivencia escolar, y asesoría y acompañamiento al docente. ORCID ID: https://orcid.org/0000-0002-5486-9241. Email:morao.noe@gmail.com 